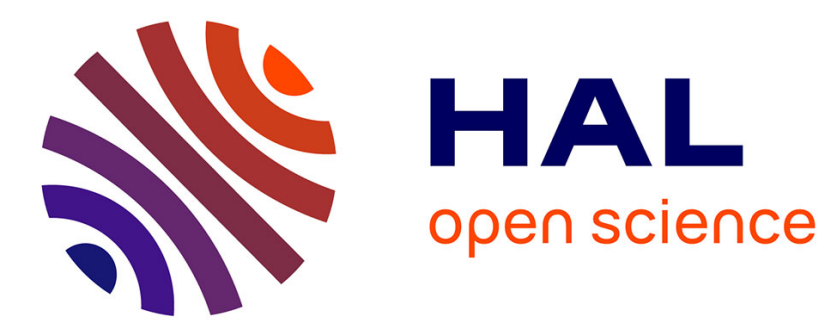

\title{
Comment se construit un mythe: la corrida en France au XXe siècle
}

\author{
Eric Baratay
}

\section{To cite this version:}

Eric Baratay. Comment se construit un mythe: la corrida en France au XXe siècle. Revue d'Histoire Moderne et Contemporaine, 1997, 44 (2), pp.307-330. hal-00485574

\section{HAL Id: hal-00485574 \\ https://univ-lyon3.hal.science/hal-00485574}

Submitted on 21 May 2010

HAL is a multi-disciplinary open access archive for the deposit and dissemination of scientific research documents, whether they are published or not. The documents may come from teaching and research institutions in France or abroad, or from public or private research centers.
L'archive ouverte pluridisciplinaire HAL, est destinée au dépôt et à la diffusion de documents scientifiques de niveau recherche, publiés ou non, émanant des établissements d'enseignement et de recherche français ou étrangers, des laboratoires publics ou privés. 


\section{COMMENT SE CONSTRUIT UN MYTHE :}

\section{LA CORRIDA EN FRANCE AU XXe SIECLE}

Depuis une trentaine d'années, la corrida bénéficie d'un indéniable engouement, caractérisé par une médiatisation croissante, par la présence aux arènes de nombreuses figures des sociétés artistique, politique, journalistique et par la diffusion d'un discours, délivré par les aficionados, repris par les médias, qui la présente souvent comme un rite d'origine antique, naturellement implanté en France méridionale, mettant en scène une tragédie fondée sur une certaine égalité des chances et le risque de mort pour le torero.

Cette image s'est imposée à l'opinion publique. Dans les pays de tauromachie, essentiellement les Landes et la Camargue étendue jusqu'au Biterrois, elle est vécue comme une réalité qui légitime le combat. Les études sociologiques montrent à quel point elle permet que la corrida soit considérée comme une composante essentielle de l'entité méridionale, jouant un rôle important dans la sociabilité régionale'.

Or, cette représentation commune ne correspond pas à la réalité. Elle constitue une légende dorée vraiment installée depuis les décennies 1950-1960, avec la légalisation de 1951, la croissance numérique des courses, la couverture médiatique. Mais elle s'est insinuée jusque dans les analyses sociologiques qui affirment souvent que la corrida n'a pu jouer une fonction sociale conséquente qu'en s'installant sur un terrain prédestiné. En s'attachant aux trois thèmes majeurs de ce discours ambiant, nous voudrions d'abord montrer qu'il s'agit bien d'un mythe, l'un des plus vivaces dans notre société contemporaine, mais surtout, cet objet est le plus important pour l'historien, retracer les modalités de sa construction en soulignant les étapes, les acteurs (notables méridionaux à la fin du XIXe siècle, cénacles intellectuels et artistiques dans l'entre-deux-guerres et la décennie 1950, journalistes et aficionados militants après 1945) et les raisons : mouvement régionaliste, rejet de l'ordre bourgeois, stratégie de défense d'une pratique minoritaire, conversion des élites à la maîtrise de la nature, évolution du statut de l'animal, etc. Car décrire les mécanismes de la constitution du mythe, c'est évoquer l'histoire de l'imaginaire occidental, des pratiques sociales et culturelles, de la relation à la nature.

\section{Les origines antiques et le culte du taureau}

L'une des idées répandues fait remonter l'origine de la corrida aux chasses préhistoriques évoquées dans l'art pariétal ou aux jeux crétois de l'époque minoenne qui se seraient diffusés sur les rivages du bassin méditerranéen ou encore aux combats dans les cirques romains, acclimatés en France méridionale dans les arènes d'Arles, de Nîmes ou de Fréjus. Depuis, l'usage de combattre des taureaux se serait pérennisé en Espagne et en France. Parallèlement, une autre opinion voudrait que la corrida ne soit que la forme moderne d'un culte ancestral du taureau dont on trouverait les premiers témoignages dans les fresques préhistoriques et qui se serait déployé dans le monde méditerranéen, de la Mésopotamie à l'Espagne en passant par l'Égypte, la Crète et l'Empire Romain investi, du ler au IVe siècle, par le mithriacisme, rival dangereux du christianisme balbutiant.

Ce culte de Mithra est souvent mentionné par les aficionados et les médias, car des vestiges de mitrhaea en Espagne et en France du sud montrent qu'il s'est implanté sur ces rivages. La filiation avec la corrida semble évidente, parce qu'il est communément analysé comme un culte du taureau même s'il existe des contradictions : certains croient qu'il s'agissait d'une dévotion rendue à un taureau mythique nommé Mithra; d'autres savent que Mithra était le nom du sacrificateur de l'animal, mais ils pensent que ce dernier fut tué avec amour et respect pour féconder la vie. Beaucoup affirment que

\footnotetext{
1 Voir les travaux de F. SAUMADE, "Mythe et histoire dans une société du spectacle tauromachique", Ethnologie française, XXI, 1991, 2, p.148-159; Des "sauvages" en Occident. Les cultures tauromachiques en Camargue et en Andalousie, M.S.H., 1994; "L'hispanité en Languedoc et Provence, une image de l'autre ", Ethnologie française, XXIV, 1994, 4, p.728-737.
} 
cette religion se traduisait par des sacrifices de taureaux et des rites de purification basés sur les gestes de Mithra : descendus dans une fosse, les fidèles recevaient le sang de l'animal qui leur apportait puissance et immortalité. La diffusion du culte dans tout l'empire romain et notamment en Ibérie s'accompagna d'un développement des combats de taureaux qui ne cessèrent plus².

Toutes ces idées sont infirmées par les connaissances actuelles. À l'apogée de la civilisation crétoise (Minoen Récent I, 1600-1450), les relations avec l'extérieur étaient centrées sur les îles de la mer Égée et ne dépassaient pas la Syrie ou l'Égypte ${ }^{3}$. En France, les arènes romaines furent détruites au moment des invasions barbares ou occupées par des habitations jusqu'au XIXe siècle (Nîmes, 1813; Arles, 1837). La transmission géographique ou historique relève à chaque fois d'une supposition, d'une confusion entre l'analogie des spectacles et la filiation réelle. D'ailleurs, l'analogie elle-même est forcée, amalgamant les différentes pratiques, supposant que tout ce qui concerne le taureau relève plus ou moins de la corrida. On sait maintenant que l'apparition en Espagne des premiers jeux taurins, chevaleresques ou populaires, se situe plus modestement autour des Xle-XIIle siècles et que la formalisation de la corrida s'effectua au XVIIle siècle à partir de certaines coutumes des abattoirs, notamment ceux de Séville ${ }^{4}$. L'écart entre le mythe et la réalité est semblable à propos du culte du taureau. Les analyses communes des peintures rupestres oublient que cet animal, en fait un bison ou un aurochs, n'est pas le seul représenté, qu'il est même moins fréquent que le cheval dans la première partie du paléolithique supérieur et pas toujours à égalité à l'époque du Magdalénien (Lascaux, Rouffignac). Pourtant, personne ne parle d'un culte des équidés. Ces idées reprennent celles de l'abbé Breuil à propos d'Altamira (où les bisons sont effectivement les plus nombreux), mais sa théorie d'une magie de la chasse et de la fécondité est rejetée par les spécialistes, depuis les années 1940-1950, au profit d'une lecture symbolique complexe. Enfin, les archéologues s'accordent à penser qu'aucun monument crétois ne témoigne d'un culte du taureau, d'un dieu-taureau, l'animal étant plutôt un attribut dont le sacrifice permettait d'offrir le sang à une divinité ou un défunt ${ }^{5}$.

L'exemple de Mithra est plus intéressant, puisqu'il concerne l'Espagne et la France. La carte des trouvailles mithriaques montre que I'lbérie fut peu concernée par cette religion de soldats, de fonctionnaires et de commerçants. Les régions privilégiées étaient la péninsule italienne, les Balkans, le limes (vallées du Rhin et du Danube) et, en moindre mesure, le couloir rhodanien, ce qui pourrait conforter la thèse usuelle. Mais cette religion de petites sociétés secrètes, réservées aux hommes, absentes des campagnes, n'a jamais fait figure de croyance universelle. La prédiction de Renan sur un monde devenu mithriaste s'il n'avait été christianisé était exagérée. Son déclin aux Ille-IVe siècles fut complet et aucun érudit ne croit en une survie clandestine au Moyen-Age.

Même si c'était le cas, cela n'arrangerait en rien la thèse commune, puisque le mithriacisme n'était pas un culte du taureau. Garant de la subsistance des créatures vivantes, Mithra fit jaillir l'eau d'un rocher et tua, d'une manière décidée et sans pitié, un taureau détenant les éléments vivifiants qu'il fallait protéger d'un ennemi invisible. Le sang de l'animal fit jaillir la végétation et nourrit les animaux. Mithra réalisait le salut bio-cosmique. Le Soleil reconnut sa suprématie et lui céda sa place; une alliance entre eux fut scellée par un repas sur la dépouille du taureau. Puis Mithra, devenu vrai Sol inuictus, monta sur le char du Soleil et rejoignit le ciel. II s'agit donc d'une sorte de culte solaire destiné, au niveau des fidèles, à exalter les vertus militaires, l'ardeur à combattre l'adversaire, et le service de la cité, comme Mithra s'était mis au service du monde. C'est pourquoi les rites d'initiation consistaient en des exercices d'endurance physique et morale qui n'avaient rien à voir avec le taureau. Si les banquets de la liturgie ordinaire réactualisaient l'alliance de Sol et de Mithra pour fortifier les membres contre les ennemis de la Vie, les animaux consommés étaient le plus souvent des volatiles, des

\footnotetext{
2 J. CONRAD, Le culte du taureau (1961), éd. 1978, p.167-175; L. LACROIX, Le taureau de combat, 1981, p.15-19; J.L. LOPEZ, dans Midi-Libre, 27.05.1993; Toros, 27.05.1995, p.26-28.

3 R. TREUIL et alii, Les civilisations égéennes du Néolithique à l'âge de bronze, P.U.F., 1989, p.316-323.

4 Voir B. BENNASSAR, Histoire de la tauromachie, Desjonquères, 1993 et E.BARATAY, E. HARDOUIN-FUGIER, La corrida, P.U.F., "Que-Sais-je?", 1995.

5 J. GARANGER (dir.), La Préhistoire dans le monde, P.U.F., 1992, p.477, 488-491; M. MASTORAKIS, M. VAN EFFENTERRE, Les Minoens, l'âge d'or de la Crête, Errance, 1991, p.187, 189.
} 
chèvres, des cervidés, des sangliers, mais très rarement des taureaux que l'on pouvait difficilement introduire et tuer dans des mithraea souvent exigus. La question se pose même sérieusement de savoir si les animaux étaient sacrifiés sur place : les contemporains ne parlent pas de liturgie sanglante, les ossements n'apportent guère de preuves, les autels ne semblent pas avoir servi à cela. La viande consommée devait souvent provenir d'animaux tués en sacrifices publics, puis vendus sur les marchés. L'immolation était symbolique. Nous sommes loin des idées usuelles qui se rapportent plutôt à Cybèle, bien que son culte ait très peu touché l'Ibérie ou la Provence ${ }^{6}$. Voyons donc comment elles germèrent dans les esprits.

C'est justement à propos de Mithra que la construction du mythe est la plus complexe et la plus significative. Les premières évocations provinrent des Félibres, soucieux de promouvoir l'autonomie, sinon politique, du moins culturelle du Midi, et elles firent d'abord appel à des explications régionalistes : dans le Poème du Rhône (1897), Mistral considéra que le taureau symbolisait la batellerie mise à mort par Mithra, l'agent du Drac, dieu de la rivière. L'idée d'un culte du taureau émergea au début du siècle chez Joseph d'Arbaud et le marquis de Baroncelli. En 1909, celui-ci voulut organiser un taurobole sur un autel dédié à Mithra, mais la menace d'excommunication de l'archevêque d'Aix l'en dissuada. Dans le poème Lou Biou (1924), il unit Apis, le Minotaure et le dieu Mithra qu'il identifiait au taureau?. L'intérêt des Félibres pour Mithra s'explique par leur volonté de dissocier la Provence, considérée comme une terre de culture antique, de la France du Nord, jugée d'origine germaine, barbare et féodale, et de la rattacher à la vieille civilisation méditerranéenne, dont justement l'un des aspects leur semblait être le culte du taureau. Le mithriacisme était ainsi transformé en ancêtre de la tauromachie, elle-même associée à l'identité méridionale comme nous le verrons plus loin.

Hormis les Félibres, dont l'audience fut limitée et l'intérêt plutôt porté sur les jeux locaux, l'idée d'un culte du taureau fut développée par Montherlant à partir des Bestiaires (1926) qu'il intégra dans un Cycle solaire comprenant les Olympiques (1924) et Pasiphaé (1936), histoire de la fille du Soleil, amoureuse d'un taureau blanc et mère du Minotaure. Comme Baroncelli, il s'inspira très librement des travaux contemporains de Franz Cumont qui révélèrent au public l'importance de Mithra et des religions orientales dans l'Empire Romain ${ }^{8}$. II bâtit ainsi une histoire du culte du taureau dont l'apogée était le mithriacisme qu'il transforma en religion universelle, accentuant les descriptions très optimistes de Cumont, oubliant que celui-ci précisait déjà que l'Ibérie n'était guère concernée. Ce n'est cependant pas chez Cumont qu'il trouva l'idée d'un amalgame entre le culte de Mithra et celui du taureau, mais chez Alfred Loisy qui publia Les mystères païens et le mystère chrétien (1919) afin de montrer que saint Paul avait donné à la cène un sens sacrificiel qu'elle n'avait pas chez les apôtres, parce qu'il était influencé par les religions orientales. Forçant la réalité pour prouver sa thèse, il affirma qu'il existait en elles une identité entre la victime et la divinité qui présidait au sacrifice : les taureaux dévorés dans les rites dionysiaques, exécutés lors des tauroboles en l'honneur de Cybèle et d'Attis, tués par Mithra, puis par ses disciples, participaient d'une même "identité substantielle et spirituelle" à Dionysos, Attis, Mithra, comme le pain et le vin étaient pour saint Paul le corps et le sang du Christ. Loisy opérait un renversement des perspectives ("le mythe de Mithra, c'est presque le mythe du taureau ") que Baroncelli et Montherlant précipitèrent en parlant de cultes du taureau.

S'appuyant sur Cumont, qui croyait que Mithra avait capturé l'animal comme les combattants des arènes romaines et qu'il existait une alliance avec le culte de Cybèle, Montherlant fit du mithriacisme le parangon des autres mystères païens et lui annexa leurs cérémonies. À l'en croire, les fidèles immolaient le taureau pour recevoir le sang en une douche génératrice (taurobole de Cybèle) et revêtaient sa peau pour s'identifier à lui et s'imbiber de ses vertus (mystères dionysiaques). II reprit

6 R. TURCAN, Mithra et le mithriacisme, Les Belles Lettres, 1993, p.35$38, \quad 43,73-85,96-104,119-120,139-145$ et Les cultes orientaux dans le monde romain, Les Belles Lettres, 1989, p.58-64.

7 Mistral : éd. 1897, chant V p.117, chant VII p.173-177; Baroncelli : J.P. BELMON, "L'invention des mythes gardians", J.N. PELEN, C. MARTEL (dir.), L'Homme et le Taureau en Provence et Languedoc, Glénat, 1990, p.137. Voir P. MARTEL, "Le Félibrige", P. NORA (dir.), Les lieux de mémoire, Gallimard, 1992, T.3, vol.2, p.567-611.

8 Textes et monuments figurés relatifs aux mystères de Mithra, 1896-1899; Les mystères de Mithra, 3e éd. 1913; Les religions orientales dans le paganisme romain, $4 \mathrm{e}$ éd. 1929. 
ainsi l'affirmation erronée de Loisy sur l'existence d'un sacrifice du taureau dans les mithraea, ce que Cumont démentait pourtant. En transformant le mithriacisme en un composé de combat et de rites, Montherlant entendait le poser en ancêtre d'une corrida qu'il découvrit en 1909 et qu'il interpréta d'une manière nouvelle. II profita d'une idée, maintenant infirmée, de Cumont (Mithra aurait tué l'animal à regret) pour soutenir que les tauroctonies étaient un mélange d'amour du taureau divin et d'obligation de le tuer pour sauver la Vie et régénérer l'officiant. Avec les mêmes gestes, le torero tue le taureau qu'il vénère pour s'imprégner de sa force et se revivifier. Montherlant métamorphosait la corrida en rite.

Elle lui servit ainsi d'intermédiaire entre le mithriacisme et le christianisme dont il prônait l'alliance. L'idée ne lui était pas spécifique. Frappés par les analogies entre le christianisme des origines et le contexte culturel de l'époque, les historiens de la fin du XIXe siècle et du premier tiers du $\mathrm{XXe}$ siècle eurent tendance à forcer les ressemblances pour montrer l'enracinement historique de la religion révélée. Cumont plaqua une lecture chrétienne sur le culte de Mithra, évoquant des rites de baptême et de communion, l'attente du salut de l'âme et du corps, la croyance au ciel et à l'enfer, et conclut sur une forte parenté entre les deux doctrines. Loisy fit de même en comparant les "cènes" mithriaque et chrétienne. On sait maintenant qu'ils eurent tort de croire aux descriptions subjectives des chrétiens et des néo-platoniciens de l'époque. Le culte de Mithra n'était pas une religion de salut individuel, mais de survie bio cosmique. Mithra n'était pas mort et ressuscité, ne s'identifiait pas à sa victime et n'avait guère à voir avec le Christ. II reste que ces interprétations permirent à Montherlant d'évoquer un parallélisme des rites et une survivance du mithriacisme dans le folklore chrétien espagnol. Ce syncrétisme se cristallisait dans la corrida (considérée comme une héritière du culte du taureau fortement teintée de catholicisme) qui devenait l'instrument d'un projet : régénérer l'Occident décadent en réimprégnant de paganisme un christianisme trop éthéré pour le revivifier, lui redonner le goût d'une existence terrestre âcre et forte ${ }^{9}$. Montherlant fut ainsi à l'origine de toute une littérature, encore d'actualité, sur le parallélisme supposé de la messe et de la corrida.

L'idée d'un culte du taureau fut vite reprise en divers cénacles. Dans les années 1930, un groupe proche du surréalisme se constitua autour de Bataille et Leiris qui découvraient la corrida et l'évoquèrent dans l'Histoire de l'oeil (1928) et le Miroir de la tauromachie (1938). Cherchant à appréhender l'homme dans sa totalité, et notamment dans sa dimension sacrée présente jusque dans les faits quotidiens, ils la parèrent d'une symbolique sacrificielle jugée évidente dans ses apparences (coïncidence avec les fêtes chrétiennes, costumes, cérémonial) et son déroulement (victoire de l'ordre sur le désordre et le maléfice). La volonté de fixer son origine les conduisit à reprendre l'idée d'un culte du taureau, d'un sacrifice régénérateur. De son côté, Picasso adopta à partir de 1928 les mythes antiques et notamment celui de Mithra dans sa nouvelle lecture. Le fait qu'une évolution semblable se produisit en Espagne montre bien que l'idée était dans l'air. La "génération 1927", formée autour de Garcia Lorca, Alberti, Bergamin, s'appuya elle-aussi sur les travaux savants pour développer l'idée d'un symbolisme sacrificiel de la corrida et d'une liaison avec le christianisme. Unamuno (1896-1936) la considéra comme une survivance d'une religion primitive. Garcia Lorca évoqua une liturgie où "comme à la messe, on adore et sacrifie un dieu ". Bergamin parla d'un mythe solaire qu'il mêla au catholicisme. Angel de Miranda écrivit une thèse sur la mythologie et les rites du taureau en Méditerranée ${ }^{10}$.

Le mythe fut donc constitué par cette génération intellectuelle de l'entre-deux-guerres. II était le fruit d'un mélange subtil entre des connaissances de l'époque soigneusement triées et de fortes imaginations. II serait possible de retracer d'une manière semblable la naissance du mythe des origines antiques, voire préhistoriques de la corrida. Au XIXe siècle, la présence des arènes rendait

9 H. MONTHERLANT, Les Bestiaires, éd. 1954, p.12, 83-85, 201-207, 214, 223, 276, 281, 293, et Coup de soleil, éd. 1976, p.229, 279; P. SIPRIOT, Montherlant sans masque, Laffont, T.1, 1975, p.78-82, T.2, 1990, p.361-369; A. LOISY, Les mystères païens et le mystère chrétien, 1919, p.195-202; F. CUMONT, Les mystères, op.cit., p.58, 136, 189-205; R. TURCAN, Mithra.., op.cit., p.109-111.

10 G. BATAILLE, "Soleil pourri" (1930), Oeuvres, Gallimard, 1970, T.1, p.231; M. LEIRIS, Miroir de la tauromachie, éd. 1981, p.58-62 et L'Age d'homme (1939), éd. Folio, p.72; Picasso, Toros y toreros, catalogue d'exposition, R.M.N., 1993, p.51, 134; J. BERGAMIN, L'art du Birlibirloque (1930), éd. 1984, p.84-89; F. GARCIA LORCA, Oeuvres, Pléiade, 1981, T.1 p.929; R. CAMBRIA, Los Toros, Madrid, Gredos, s.d., p.70, 130-140. 
déjà populaire l'idée d'une création romaine. La découverte de jeux taurins dans la Thessalie antique, les fouilles d'Evans à Cnossos (1900-1941) avec la mise à jour de fresques représentant des courses, ses travaux (1906-1936) évoquant le palais de Minos ainsi qu'une thalassocratie crétoise, ceux de l'abbé Breuil (1906) à propos de la grotte préhistorique d'Altamira permirent d'approfondir la généalogie $^{11}$. Le comte de Las Navas (1855-1935) établit ainsi une liaison entre les chasses préhistoriques à l'aurochs et la corrida. Dès 1926, Montherlant affirma que les Thessaliens et les Crétois apportèrent leurs jeux dans le Midi. II projeta décrire un roman sur les Crétois, puis publia Pasiphaé. C'est justement dans ces années 1930 que Picasso mêla pour la première fois le mythe du Minotaure, librement interprété, à la corrida (Minotauromachie, 1935) ${ }^{12}$.

L'évolution des connaissances aurait peut-être pu freiner ces constructions intellectuelles. Dès le milieu du siècle, les archéologues savaient que la thalassocratie crétoise se cantonnait à la mer Égée et qu'on ne pouvait parler d'un culte du taureau. Dans les années 1960-1970, les erreurs de Cumont furent corrigées et les interprétations de Loisy sévèrement réprouvées ${ }^{13}$. Mais cela n'empêcha pas la diffusion du mythe dont on peut suivre aisément les étapes. Dans les décennies 1940-1960, il fut d'abord véhiculé par des écrivains (Prévert, Cocteau, Druon..) disciples de la génération précédente, puis par des ethnologues attirés aux arènes par la renommée croissante de Bataille et de Leiris. Plus sensibles, par leur formation, aux analogies des rites, aux structures mentales intemporelles qu'à la réalité de la filiation, ces derniers le systématisèrent et l'accentuèrent. L'exemple le plus significatif est celui de l'anthropologue américain J. Conrad qui synthétisa tous les topos, notamment ceux de Montherlant, dans Le culte du taureau (1961), devenu la bible des aficionados. Alors qu'au même moment, les archéologues se demandaient prudemment si les Crétois avaient quelques relations avec la Sicile, une idée abandonnée depuis, il les faisait triomphalement débarquer en Ibérie, puis dans les îles britanniques pour justifier l'existence d'un culte celtique du taureau ! ${ }^{14}$. Ce discours se diffusa parmi les toreros célèbres, tel Dominguin qui fréquentait Cocteau et Picasso, et parmi les aficionados grâce à ces intermédiaires culturels que furent les écrivains chroniqueurs taurins, par exemple Jean Cau ou Jean-Marie Magnan, ami de Cocteau. Bien que quelques-uns ne l'acceptent pas, il est largement adopté depuis la décennie 1970 (des éleveurs camarguais nomment certains de leurs taureaux du nom de Mithra) et se répand dans l'opinion publique grâce aux médias ${ }^{15}$.

Comment expliquer ce développement continu du mythe ? Difficulté à appréhender une science de plus en plus complexe et parcellisée ? Sans doute. Force poétique et intellectuelle des oeuvres de Montherlant, Picasso, Bataille, Leiris ? Sûrement. Mais il s'agit aussi d'une impossibilité croissante, à mesure qu'il se diffusait et s'imposait comme une réalité, à changer cet imaginaire qui servait bien les intérêts des aficionados dont l'un des buts au XXe siècle fut d'imposer la notion d'une tradition de la corrida. L'idée apparut dans les années 1890 pour contrer l'opposition des mouvements de protection soutenus par les milieux intellectuels et politiques. Elle obtint sa reconnaissance juridique avec la loi de 1951, autorisant justement la corrida dans les régions de tradition ininterrompue. Elle bénéficia ensuite d'un discours amplifié afin de présenter cette loi comme une simple mesure de justice alors que les opposants parlent toujours d'une exception inique à la loi Grammont ${ }^{16}$. Depuis les

11 E. BEURIER, "Les courses de taureaux chez les grecs et les romains", Mémoire de la Société des Antiquaires de France, 1887, p.73; A. EVANS, The prehistoric tombs of Knossos, 1906 et The Palace of Minos, 1921-1936; H. BREUIL, E. CARTAILHAC, La caverne d'Altamira, 1906, du même, Les peintures rupestres schématiques de la Péninsule ibérique, 1933.

12 P. SIPRIOT, op.cit., T.2 p.360-361; Picasso.., op.cit., p.147.

13 F. MATZ, Le monde égéen, 1956, p.73-79; M. VERMASEREN, Mithra, ce dieu mystérieux, 1960.

14 J. PREVERT, Spectacle (1951), éd. Folio, p.223; J. COCTEAU, La corrida du premier Mai (1957), éd. 1988, p.57; J. CONRAD, op.cit. , p.176-182.

15 Dominguin dans P. PICASSO, Toros y toreros, éd. 1980, p.10-11; J. CAU, Toros, 1973, p.14; exemples parmi les aficionados, C. PELLETIER, L'heure de la corrida, 1992, p.16-18, et les médias : FR3, Soir 3, 3 avr. 1994. Les doutes : B. BENNASSAR, op.cit., p.12.

16 Journal Officiel, A.N., annexes, 1900, p.1211; J. PERRIN dans Le Monde, 20.03.1988, p.12. Voir A. LAFRONT, Histoire de la corrida en France, Julliard, 1977. 
années 1970, cette stratégie est facilitée par l'évolution de la société française qui valorise les traditions et les justifie quasiment par elles-mêmes. D'où la volonté des aficionados de lier la corrida au passé le plus lointain, de véhiculer un mythe devenu plus important que la vérité. II s'agit de transformer le combat en une coutume millénaire, voire en une caractéristique originelle et essentielle de l'humanité17.

\section{La France méridionale, une terre naturelle de la corrida}

Ce mythe intervient souvent en complément chronologique du premier, mais il est aussi repris par ceux qui ne croient pas aux origines antiques. II repose sur l'idée qu'il existe en Provence et dans les Landes une vieille tradition taurine, incarnée par les courses camarguaise et landaise, responsable d'une culture tauromachique profondément ancrée, quasi-consubstantielle au peuple de ces régions : " Dans le Midi taurin, déclarait le président Doumergue, ardent défenseur de la corrida dans les années 1900-1920, la passion des taureaux à des racines plus profondes qu'en Espagne même ". Dès lors, la corrida, introduite à partir de 1852, ne peut être considérée comme un élément étranger, plaqué sur la culture méridionale. Elle fut "appelée " par la tradition taurine et s'enracina rapidement dans un territoire prêt à l'accueillir. Elle appartient au peuple du Midi "par les profondeurs " affirma Montherlant en 1926. Cette même tradition expliquerait le maintien naturel, obligatoire des corridas jusqu'à nos jours. Par contre, son absence au nord de la France vouait à l'échec les tentatives d'introduction qui eurent lieu dans les années 1860-1940. En ce sens, la loi de 1951 ne fit que transcrire la réalité en acceptant la tradition taurine du Midi, en reconnaissant l'impossibilité d'une implantation contre-nature en France du Nord ${ }^{18}$. L'explication est en réalité très réductrice. Elle est uniquement fondée sur l'analogie géographique actuelle des jeux locaux et des corridas. Elle ne tient pas compte de la complexité du processus historique où plusieurs facteurs interviennent à des intensités variables à l'échelle locale. II s'agit d'une reconstruction du passé destinée à justifier les convictions présentes. Elle est devenue commune parmi les aficionados et se voit véhiculée par les médias, parce que les autres causes sont oubliées.

Ce mythe est basé sur le postulat qu'il n'existerait des jeux taurins que dans le Midi. En réalité, les courses landaises et provençales ne furent formalisées que récemment, aux XIXe-XXe siècles. Auparavant, il existait divers types de jeux, organisés lors des fêtes locales : combats de taureaux contre des chiens ou des bêtes sauvages; courses de taureaux dans les rues en les excitant avec des bâtons ou des aiguillons et en esquivant leurs charges; " taureau à la corde " en Provence, où l'animal, dirigé par une corde, était soumis à des sévices avant d'être abattu, etc. Or cette tauromachie populaire était répandue en d'autres régions. À l'époque moderne, des enciré (course du taureau au milieu d'une foule selon un itinéraire déterminé) avaient lieu à Stamford et Tutbury en Angleterre et des combats de chiens et de taureaux à Birmingham, Bristol ou Venise. En France, des courses se déroulaient régulièrement à Autun au XVIle siècle et des combats d'animaux (notamment taureaux contre chiens) à Paris, Lyon, Rouen, etc., aux XVIIle-XIXe siècles. Tout cela disparut peu à peu, au plus tard à la fin du XIXe siècle, sans que ces régions aient accueilli la corrida. En fait, le mythe repose sur une confusion entre l'état de la recherche et la réalité : on connaît plus d'exemples provenant du Sud-Ouest ou de la Provence, parce que les érudits aficionados sont allés chercher leurs traces dans les archives afin d'étayer la thèse commune, ce que personne n'a fait ailleurs ${ }^{19}$.

D'autre part, l'évolution des courses locales vers une moindre violence ne facilita pas la jonction avec la corrida. La première moitié du XIXe siècle fut marquée par la répulsion croissante d'une partie des élites régionales et parisiennes envers la violence des courses, notamment les

17 J.L. LOPEZ dans Midi libre, 27.05.1993.

18 L. FEUILLADE, Chroniques taurines, 1899-1907, 1988, p.120; H. MONTHERLANT, Les Bestiaires, op.cit., p.11, 12 (Doumergue); D. GATUMEL, Tauromachie, société et mentalité, Maîtrise, Montpellier, 1980, p.96. Idée reprise par F. SAUMADE, "L'hispanité..", art.cit., p.728-729.

19 Exemples étrangers : B. BENNASSAR, op.cit., p.12; Toros, 24.04.1988; Autun : P. DUPUY, La tauromachie en France en 1900, 1982, p.124; Paris et Rouen : J. DULAURE, Histoire physique, civile et morale de Paris, 1824, p.417, M. LE GRAND, Les courses de taureaux dans le Sud-Ouest de la France jusqu'au début du XIXe siècle, 1934, p.77; Lyon : affiche de 1823, A.D. Rhône. Sur les érudits, voir l'exemple cité de M. Le Grand. 
traitements infligés aux bêtes ${ }^{20}$. Si elles ne purent obtenir leur suppression à cause de la résistance des populations, leur opposition obligea tout de même à une transformation des pratiques : les courses de rues furent refoulées dans des espaces clos; des municipalités recommandèrent de ne pas maltraiter les taureaux après le vote de la loi Grammont (1850) qui punissait d'une amende l'auteur de sévices publics sur les animaux domestiques; en Provence, le spectacle plus policé de la course à la cocarde, où des hommes tentaient d'arracher cette enseigne du frontal du taureau, se développa après la Révolution ${ }^{21}$. C'est pourquoi, l'accueil de la corrida ne fut pas aussi spontané qu'on le prétend. Arles, la capitale de la course camarguaise, ne connut pas de corridas avec mise à mort avant 1892 (1863 à Nîmes). Elle n'en organisa ensuite qu'une par an, avec une interruption à la fin du siècle, puis de 1923 à 1931. En 1884, une agitation populaire, née à l'annonce d'une interdiction gouvernementale des courses, s'arrêta dès qu'on apprit qu'elle ne concernait que les espagnoles. À cette occasion trois députés du Midi montèrent à la tribune de l'Assemblée Nationale pour refuser l'idée d'une liaison entre les jeux locaux et les corridas qu'ils condamnèrent vigoureusement. En 1900, le journal La Gironde démentit l'idée que celles-ci fissent partie de la tauromachie traditionnelle et $46 \%$ des députés des Bouches-du-Rhône, du Vaucluse, de la Haute-Garonne, du Gers et de la Gironde votèrent en faveur de l'examen en urgence d'une proposition de loi les interdisant ${ }^{22}$.

En réalité leur introduction fut laborieuse, en priorité à cause de la réserve du public. Lors des trois corridas données en 1853 à Bayonne (première ville à les organiser), les spectateurs désertèrent les gradins dès le second jour. En 1859, l'assistance diminua de moitié lors de la deuxième course et la troisième fut annulée. Même scénario à Mérignac en 1853. Même échec à Nîmes en 1863. Après leurs expériences décevantes de courses mixtes en 1861, Saint-Sever se replia sur les landaises et Dax oublia les corridas jusqu'en 1878. À Bayonne, l'impresario résilia son bail en 1860, son successeur fit de même en 1862. Peu enclins à admettre cette réserve, beaucoup d'aficionados expliquent ces difficultés par la médiocrité des combats, mais le public de l'époque n'avait pas l'expérience pour en juger et cela supposerait de la part des organisateurs un acharnement louable, mais suspect à faire faillite ! En fait, la population méridionale ne se distinguait guère de celle du nord où les échecs furent nombreux une fois passée l'attrait de l'exotisme, une situation cette fois reconnue par tous, car elle semble prouver l'incompatibilité géographique ${ }^{23}$.

Les difficultés de l'introduction furent aussi le fait d'une vive opposition, y compris régionale, nous l'avons vu. Le monde intellectuel, les évêques locaux (Aire, Nîmes), la S.P.A. condamnèrent les corridas et obtinrent l'intervention des pouvoirs publics ${ }^{24}$. Les municipalités ne résistèrent guère et respectèrent dans l'ensemble les interdictions répétées de 1881, 1884, 1886, même si des corridas intégrales furent organisées à la sauvette à Nîmes ou dans les Landes. En fait, la volonté des organisateurs de ne pas choquer et de prouver leur respect de la loi Grammont les conduisit le plus souvent à se servir, tel un cheval de Troie, des courses locales pour monter des spectacles mixtes qui se maintinrent jusque dans le premier tiers du XXe siècle : courses burlesques, hispano-landaises,

20 Exemples du ministre de la police en 1801, dans M. LE GRAND, idem, p.101, de A.L. MILLIN, Voyage dans les départements du Midi de la France, 1807-1811, dans J.N. PELEN, C. MARTEL (dir.), op.cit., p.221, du comte de Villeneuve, dans Moeurs, usages, coutumes et langage des provençaux, éd. 1972 , p.105.

21 E. DURET, "La course camarguaise, aspects historiques", J.N. PELEN, C. MARTEL (dir.), idem, p.71-77; F. SAUMADE, "Mythe..", art.cit., p.152; M. LE GRAND, ibid., p.74-87.

22 B. BENNASSAR, op.cit., p.190-191 (Arles); Journal Officiel, A.N. débats, 22.06.1884, p.1427-1430 et 15.01.1900, p.50, A.N. annexes, 08.06.1900, p.1209. Carte du vote de 1900 dans E. BARATAY, E. HARDOUIN-FUGIER, op.cit., p.52.

23 M. ODUAGA ZOLARDE, Les courses de taureaux expliquées, (1854), éd. 1988, p.55; A. LAFRONT, op.cit., p.29-30, 38, 42, 44, qui reprend l'explication de la médiocrité. Au Nord, voir les exemples de Paris, A. LAFRONT, idem, p. 57-62, et Roubaix, P. DUPUY, op.cit., p.30.

24 Voir Le Temps, 11.08.1896, 96.06.1900; SEVERINE, Pages rouges, 1883, p.93; H. PLANTIER (Nîmes), Instructions, lettres pastorales et mandements, 1867, T.1 p.231-249; Bulletin de la S.P.A., fév. 1894, p.105. 
hispano-provençales où les acteurs multipliaient les passes, les sauts, les poses de banderilles ou de cocardes, avec quelquefois des piques, mais sans l'estocade. Si les jeux locaux servirent de niche écologique aux corridas, ce fut plus par nécessité que par attrait. D'ailleurs, après une forte liaison entre les décennies 1890 et 1920, un divorce s'effectua, laissant la place à des publics, des clubs, des journaux différents. Les difficultés furent donc fortes jusqu'aux années 1890. 1852-1891 : environ quarante ans d'incertitude; on ne peut parler d'une arrivée logique et naturelle dans les pays taurins. À Nîmes, par exemple, les corridas ne furent présentes que neuf années sur trente-huit (1853-1891 ${ }^{25}$.

Enfin, les aficionados érudits savent que les introducteurs de la corrida furent souvent d'origine espagnole. Profitant du développement des réseaux routier puis ferré, ils mirent sur pied une véritable entreprise d'exportation des hommes (peu nombreux, les toreros français n'eurent que des carrières médiocres) et des bêtes qui supplantèrent rapidement le bétail local, jugé inapproprié. Or ces impresarios et ces toreros ne firent aucune différence entre les régions taurines et les autres. Ils avaient au contraire la volonté de profiter partout de l'attrait (pourtant relatif) pour le folklore et l'exotisme. Si les courses mixtes, à base de corrida, se développèrent dans les contrées les plus proches des Pyrénées dès la décennie 1850, les tentatives d'implantation plus au nord se multiplièrent les années suivantes. Pablo Mesa organisa des spectacles à Périgueux, Agen, Poitiers en 1866, au Havre en 1868. Des bruits d'introduction à Paris circulèrent dès 1855 et l'affaire fut effective en 1865. Le torero Mazzantini oeuvra pour la construction d'une arène au bois de Boulogne en 1889 et, en 1900, une société anonyme étudia la possibilité d'en gérer une avec un bail de trente ans. La période 1890-1910 fut celle d'une poussée le long de l'Atlantique (Rochefort-sur-Mer 1897, Nantes 1906, Saint-Malo 1912, etc.) et à l'Est (Vichy 1892, Lyon 1894, Roubaix 1899, Reims 1900, Autun 1913, etc.). Dans l'entre-deux-guerres, les espoirs ne cessèrent pas, entretenus cette fois par les aficionados français : Paris en 1926, Royan et Quimper en 1934, Limoges en 1935, etc. (le plus souvent il s'agissait toujours de courses mixtes ou de corridas édulcorées). En 1949, ils croyaient encore qu'une implantation durable dans la capitale était possible ${ }^{26}$. La loi de 1951 ruina ces espérances et créa une distinction entre les régions à tradition taurine et les autres, donnant une reconnaissance juridique à un mythe progressivement construit.

Celui-ci émergea dans la seconde moitié du XIXe siècle pour des raisons de stratégie. Face à I'hostilité des élites, notamment parisiennes, les aficionados, en particulier les impresarios et les directeurs d'arènes, développèrent une politique d'investissement des jeux locaux, concrétisée par les courses mixtes qui permirent de contourner les oppositions et de s'installer : "nous espérons, déclara en 1889 Prosper Séris, un des premiers critiques landais, que la faveur que le public leur accorde (aux hispano-landaises) nous achemine peu à peu vers le but que doivent poursuivre les vrais aficionados : la corrida espagnole pure ". Mais l'investissement fut aussi mental avec la proclamation incessante d'une liaison historique, technique, culturelle entre les courses régionales et la corrida au nom de la fraternité latine des peuples du sud ou de l'ancestrale passion taurine des deux pays ${ }^{27}$. Cette politique fut sensible dans la création des premiers journaux (Le Picador et Le Toréador en 1888, Le Torero en 1889) et clubs taurins (Nîmes en 1896, Arles en 1897..) par une bourgeoisie urbaine locale qui s'intéressait en priorité à la défense de la corrida sous le couvert d'une passion proclamée pour les jeux locaux, véritables étendards de la tradition en réalité peu considérés ${ }^{28}$. Elle trouva tout son intérêt lorsqu'il s'agit de contrer les interdictions gouvernementales en 1884, 1886, 1894-1895 ou le projet de

25 On trouvera un récit honnête, mais orienté, des années 1852-1891 dans A. LAFRONT, op.cit.; sur le divorce des courses, voir J.N. PELEN, C.MARTEL (dir.), op.cit. p.61-80; D. GATUMEL, op.cit., diagrammes (Nîmes).

26 Sur les tentatives : A. LAFRONT, idem; la stratégie d'exportation : B. BENNASSAR, op.cit. , p.139; Paris en 1855, LAGARDE-MONTLEZUN, Rapport sur la question des combats de taureaux, 1855, p.2, en 1900, Journal Officiel, A.N. débats, 15.01.1900, p.34, et en 1926, P. SIPRIOT, op.cit., T.2 p.360; Documentation catholique, 1935, col.1469 (Quimper); Semaine religieuse de Limoges, 16.06.1935; Le Monde, 08.05.1949 (O. Merlin).

27 Séris cité par A. LAFRONT, op.cit., p.46; L. FEUILLADE, op.cit. , p.128; M. ODUAGA-ZOLARDE, op.cit., p.50.

28 Voir A. CHAMAND, "Histoire et place des clubs taurins" et N. PEYRUSSAN, "Histoire, contenu et enjeux de la presse taurine", J.N. PELEN, C. MARTEL (dir.), op.cit., p.81-96, 97-112; D. GATUMEL, op.cit. , p.146-147. 
loi de 1900. À chaque fois les aficionados prétendirent que toutes les courses étaient concernées et que les traditions étaient menacées, alors que le gouvernement et la S.P.A. s'évertuaient à préciser le contraire (sauf en 1895) ${ }^{29}$. Cette stratégie échoua en 1884, nous l'avons vu, mais elle réussit en 1894 quand le directeur des arènes de Nîmes parvint à rassembler un large public pour une grande corrida de protestation. Elle aboutit aussi en 1900 lorsque les clubs taurins recueillirent de nombreuses signatures au bas de leurs pétitions contre le projet de loi. Celle d'Arles, par exemple, affirmait que toutes les courses étaient en danger et que la corrida, implantée récemment, mais apprise rapidement grâce à "nos connaissances spéciales, innées chez nous, du toro de combat", était le jeu favori des populations. Cette politique triompha encore en 1921 : les dirigeants des clubs nîmois, en particulier l'avocat Bernard de Montaud, obtinrent le concours du marquis de Baroncelli, législateur et promoteur des courses camarguaises, pour la défense de la corrida ${ }^{30}$. Tout cela au moment même où nombre d'aficionados réclamaient l'abandon des courses mixtes, un détachement vis-à-vis des jeux locaux jugés charmants, mais sans grandeur, et le développement des corridas intégrales ${ }^{31}$.

Le discours sur la tradition avait une autre fonction politique. Les oppositions les plus vives eurent lieu lors des tentatives d'implantation au nord de la France, notamment en région parisienne, parce qu'elles semblaient narguer le gouvernement et la S.P.A.. Elles suscitèrent ainsi le projet de loi de 1900, mais la thèse taurine fut l'un des moyens employés pour désamorcer le danger. Les municipalités et les parlementaires méridionaux qui soutenaient la corrida arguèrent du fait que le Midi ne pouvait être privé d'un pan de sa culture, mais ils reconnurent le caractère incongru et malheureux des tentatives effectuées au Nord. II ne faut cependant pas conclure à une simple duplicité, même si elle existait. Cette attitude reflète aussi une division parmi les aficionados : alors que d'aucuns (toreros, impresarios, militants septentrionaux..) penchaient pour une extension maximale, d'autres prônaient un repli prudent sur les bastions méridionaux que le mythe de la tradition devait rendre inexpugnables ${ }^{32}$. Car l'idée commençait à obtenir quelques crédits dans l'opinion publique. Elle permit d'enterrer le projet de 1900, puis celui de 1911. Lorsqu'en 1951, des parlementaires demandèrent une autorisation de la corrida sans limites géographiques, le gouvernement imposa un compromis qui n'était que la traduction juridique du mythe et l'aboutissement d'une stratégie de 70 ans, puisqu'il ne distinguait pas la course espagnole des jeux locaux et qu'il acceptait la théorie d'une coutume ininterrompue en certaines régions ${ }^{33}$.

Cependant, le mythe n'était pas crédible sans le développement d'un public méridional. Celuici n'est pas né spontanément sous l'effet d'un attrait inné pour la corrida. II émergea dans les années 1890-1900, comme le révèle la création des journaux, des clubs et de l'Union Tauromachique de France en 1901, après ce qui peut finalement apparaître comme une époque de gestation. Ce public de militants, dont les cadres appartenaient à la bourgeoisie locale (notables urbains, journalistes, éleveurs ${ }^{34}$ ), fut très actif pour défendre la corrida et l'imposer dans sa version intégrale. II était néanmoins restreint, estimé par A. Lafront à 2500 personnes pour le Sud-Ouest vers 1930, et n'existait pas seulement dans ces régions, mais à Paris par exemple (journaux Le Torero et Paris-Toros en 1889, club Le Toro au début du siècle $)^{35}$. En réalité, le facteur décisif pour le développement d'une assistance conséquente fut l'immigration espagnole. Reconnu comme une évidence pour les autres

29 Journal Officiel, A.N. débats, 22.06.1884, p.1430; Bulletin de la S.P.A., fév.1895, p.39, 144, 324; Journal Officiel, A.N., 1900, annexe 1691.

30 Bulletin de la S.P.A., fév. 1895, p.147 (Nîmes), 1900, p.134 (Arles); Protestation du club taurin d'Arles contre le projet de loi Bertrand, dans J.L. LOPEZ, Corrida oui, corrida non, 1977, p.25-28; Toros, 27.11.1991, p.9-11 (Nîmes en 1921).

31 L. FEUILLADE, op.cit., p.62, 108; H. MONTHERLANT dans L'Auto, 09.02 .1933$.

32 M. ODUAGA-ZOLARDE, op.cit. , p.50; H. MONTHERLANT, idem.

33 Journal Officiel, A.N. annexes, 08.06.1900, p.1209; Bulletin de la S.P.A., 1901, p.228; Journal Officiel, A.N. annexes, 1950, p.821, 1637, 2405; Journal Officiel, C.R., débats, 1951, p.1072-1073.

34 Les origines sociologiques des aficionados locaux, notamment des diri geants, ne sont connues que d'une manière impressionniste. Une étude pré cise reste à faire sur ce point. 
pays de corrida (Amérique Latine), cet élément fut toujours occulté par la littérature taurine française parce que ces migrants, encore mal intégrés, appartenaient rarement à l'élite dirigeante de l'aficion et parce qu'il fallait mettre au premier plan l'idée d'un besoin local inné. Or l'influence de la communauté d'origine espagnole est manifeste : elle fut en grande partie à l'origine de l'introduction de la corrida; elle joua un rôle important dans l'initiation des autochtones (on connaît l'influence de Picasso sur Braque, Cocteau, Desnos..); elle constitua un public important et régulier dans le Midi. Car à côté d'une migration politique commencée en 1814, mais dispersée géographiquement pour des raisons de sécurité, un exode économique se développa au XIXe siècle. Venus chercher une vie meilleure, artisans et cultivateurs s'installèrent en priorité au sud de la Garonne et du Massif Central, dans la vallée du Rhône et en Provence. Nîmes, capitale taurine dès le début du XXe siècle, était un point fort de cette immigration. En majorité issues des contrées méditerranéennes ou méridionales de l'Espagne, ces populations étaient souvent attachées à la corrida, image d'une hispanité perdue, et elles renforcèrent le public local avant que les touristes et la jet society parisienne ne vinrent compléter les gradins à partir des années 1950. Ainsi l'aficion gardoise actuelle, décrite par F. Saumade, se partage entre une bourgeoisie urbaine férue d'exotisme et des milieux populaires d'origine espagnole ${ }^{36}$

On objectera qu'il existait une immigration importante en direction des régions parisienne et lyonnaise. En 1931, les trois-quarts des migrants étaient installés dans une zone allant de la Gironde aux Bouches-du-Rhône, mais aussi dans les départements de la Seine, du Rhône et de la Loire. Des témoignages montrent qu'ils constituaient, dès la fin du XIXe siècle, une bonne partie de l'aficion parisienne qui militait pour une implantation de la corrida dans la capitale ${ }^{37}$. Cependant, le flux le plus important en ces contrées eut lieu dans les années 1950-1960, donc après la loi de 1951, et la pression fut toujours contenue par l'opposition de la S.P.A., soutenue par les gouvernements, ainsi que par l'absence d'un sentiment régionaliste ${ }^{38}$.

Car celui-ci constitue le troisième facteur du mythe taurin, permettant non pas de l'émettre ou de l'accréditer, mais de le faire accepter par les populations méridionales. La coordination des aficionados à partir de la fin des années 1880 facilita les pressions sur les députés, les sénateurs et surtout les maires, désormais élus par les conseils municipaux et non plus nommés par le gouvernement depuis les lois de 1882 et 1884 . À partir de 1891 dans les Landes et 1892 dans le Gard, les corridas se multiplièrent. Les municipalités feignirent d'ignorer la loi Grammont, tournèrent les interdictions, organisèrent des corridas de protestation (Nîmes et Dax en 1894). Elles profitèrent des tergiversations des autorités qui balançaient, l'instabilité ministérielle aidant, entre la fermeté et la tentation du laisser-faire pour ne pas froisser des populations en majorité républicaines à propos d'une question jugée mineure. La fronde fut menée au nom des libertés municipales et de celles du "peuple du Midi ". L'argument n'était pas nouveau. II fut utilisé au XVIIle siècle et dans la première moitié du XIXe siècle pour la défense des courses locales. II s'insérait dans une attitude générale de réserve visà-vis du pouvoir central, traduisant une intégration plus lente et plus tardive à un territoire français en voie d'unification. Les élites du pays reportant leur prévention sur les corridas, il déclina dans les années 1850-1860, mais il revint en force dans la décennie 1890 pour défendre ces dernières ${ }^{39}$. II fut alors sans cesse repris par les aficionados, parce qu'il leur donnait localement un poids politique qu'ils n'avaient pas au niveau national ${ }^{40}$.

35 A. LAFRONT, op.cit. , p.136; D. GATUMEL, op.cit., p.147-148; H. MONTHERLANT, "Taurinus furor", Coups.., op.cit., p.255.

36 Des Sauvages.., op.cit., p.69 et "L'hispanité..", art.cit.

37 Le Temps, 06.06.1900; L. FEUILLADE, op.cit., p.71; A. LAFRONT, op.cit., , p.53.

38 G. HERMET, Les Espagnols en France : immigration et culture, Ed. Ouvrières, 1967, p.24-36; B. BENNASSAR (dir.), Histoire des espagnols, Laffont, 1992, p.1013-1038.

39 Voir les journaux Le Temps, 13 et 16.10.1894, L'lllustration, 23.02.1895 et A. LAFRONT, op.cit. . Sur I'histoire du thème : E. DURET, art. cit., p.70; M. LE GRAND, op.cit. . Sur le régionalisme : M. AGULHON. "Conscience nationale et conscience régionale en France de 1815 à nos jours", Histoire vagabonde, Gallimard, 1988, T.2 p.144-174.

40 L. DUPUY, A la S.P.D.A. Protec-Toros, 1895, p.5; chansons "La Marseil - 
Ce discours sur les libertés prit une ampleur considérable en ces années 1890-1910, car il intervenait en plein apogée du Félibrige. Constitué en réaction à l'insertion du Midi dans l'espace français, ce mouvement traduisait le malaise des classes moyennes, les premières à bénéficier de la francisation, face à la régression de la culture traditionnelle. Mais les espoirs d'autonomie furent balayés après 1870 par la grande vague patriotique que connut la France et le mouvement se replia dès la décennie 1880 sur la défense des libertés et des coutumes régionales, notamment la langue, les costumes, auxquels s'adjoignirent progressivement les courses de taureaux. Frédéric Mistral, fondateur du Félibrige, ne les goûtait guère, même les locales, et ne leur réserva qu'une maigre place dans ses oeuvres. Pourtant sa participation à la corrida nîmoise de protestation en 1894 révèle une première évolution. II déclara préférer les jeux provençaux et montra qu'il n'était pas dupe de l'amalgame opéré par les aficionados. Néanmoins, il accepta d'inclure la corrida dans la défense des traditions régionales et des libertés municipales : "Sans être partisan personnellement des courses espagnoles, j'aime tout ce qu'aiment mes compatriotes ". Une position précisée par Emile Ripert : " bien qu'il se souciât assez peu de se faire, comme il le disait, "le porte-drapeau de la tauromachie espagnole ", il n'avait pu se défendre de présider une manifestation où semblait engager la défense des libertés méridionales ". L'intégration de la corrida s'acheva trente ans plus tard avec le marquis de Baroncelli qui considéra la Camargue comme le sanctuaire de la Provence épargnée par le progrès destructeur, se fit manadier (éleveur de taureaux) en son sein et promut les courses au rang de symbole de l'identité méridionale. II contribua à la formalisation de la tauromachie camarguaise et à sa séparation d'avec la corrida, mais il n'hésita pas à défendre celle-ci. En 1921 par exemple, il signa avec les dirigeants de l'aficion nîmoise une déclaration commune qui arguait du sacrifice de la liberté méridionale à la construction de la nation pour revendiquer le droit à la langue et aux courses de taureaux, sans qu'il soit fait une distinction entre ces dernières ${ }^{41}$.

Si l'immigration et le régionalisme furent les grands vecteurs de l'implantation de la corrida en France, contraignant définitivement les gouvernements au laisser-faire, ils permirent aussi de déployer un mythe construit pour des raisons de stratégie et de l'imposer à l'opinion publique. En ce sens, la loi de 1951 entérina ces deux aspects, la victoire du Midi et la réussite médiatique du mythe (même si quelques aficionados érudits doutent de sa réalité), puisque tous les députés et sénateurs méridionaux la votèrent, contrairement au projet de 1900, tandis qu'elle recueillit une large majorité dans les régions septentrionales ${ }^{42}$.

\section{La tragédie de la mort}

Depuis les années 1930-1950, un discours sur l'omniprésence de la mort pour l'homme s'est progressivement développé. De Joseph Peyré (Sang et Lumières, prix Goncourt 1935) à Pierre Mialane (La peur, la beauté, la mort, 1981) en passant par Alain Borne (Vive la mort, 1960), il figure dans la plupart des textes taurins jusqu'à devenir un passage obligé dans les décennies 1970-1990. La corrida est présentée comme une tragédie où la mort rôde et choisit à son gré l'animal, fréquemment, ou l'homme, bien souvent. La peur, les blessures, la mort, une certaine égalité des chances sont sans cesse évoquées. Joseph Peyré et Simon Casas insistent sur l'angoisse du torero avant le combat et sa peur quotidienne même dans les jours ordinaires. Mialane affirme que les matadors ont la nécessité de mettre leur vie en péril, que le paseo représente un défilé vers la mort aussi éprouvant que le combat, que celui-ci est tragique au regard des "centaines de "soldats inconnus " de l'art taurin ". Fornairon pense que la grandeur de la corrida réside dans la mort obligée de l'animal et celle, éventuelle, de l'homme. Jean Cau considère que les toreros mettent de la beauté sur le tragique de la condition humaine en s'enlaçant à la mort et en s'en défaisant, mais pas toujours. Jean-Pierre Darracq soutient que "L'égalité des chances entre l'homme et la bête (...) constitue la justification, la seule, du drame taurin". Allant plus loin, Bourdin et Mialane écrivent que "l'hypothèque

\footnotetext{
laise tauromachique" et "Nîmes bouge" dans J.L. LOPEZ, op.cit., p.70-71.

41 La Revue Félibréenne, 1894, p.183-184; Le Journal, 15.10.1894 et La Libre Parole, 22.07.1896, cités dans Toros, 06.08.1989, p.31; Avec Mistral sur les routes de Provence, 1931, p.71; texte de 1921 dans J.L. LOPEZ, op.cit., p.77. Sur le Félibrige: P. MARTEL, art. cit.; sur l'intégration des courses : J.P. BELMON, art.cit., p.135-140.
}

42 Les doutes : B. BENNASSAR, op.cit., p.190. Carte du vote des sénateurs en 1951 dans E. BARATAY, E. HARDOUIN-FUGIER, op.cit. , p.52. 
ne sera jamais levée (...) Un homme va aller affronter un taureau et va le tuer. Ou bien ce sera le contraire. L'insupportable inverse ! (...) Et puis, qu'on ne me raconte pas d'histoires avec ce poncif de fin de siècle : " l'égalité des chances ! ", si le torero a bien une épée, son adversaire en a deux! "43.

Tout cela aurait étonné les aficionados du XIXe siècle et de la première moitié du XXe siècle, habitués qu'ils étaient à mettre l'accent sur le courage, le sang-froid, la force du matador. La corrida ne leur semblait pas une tragédie, mais un combat mêlant la technique, l'art et l'intelligence. Pour le poète provençal Laurent Tailhade, elle permettait de montrer les qualités viriles de l'homme. Pour OduagaZolarde, auteur du premier manuel taurin français en 1854, elle distinguait I'homme des bêtes et assurait sa supériorité sur elles. Elle constituait la plus ingénieuse mise en scène d'une entreprise millénaire de domination sur la nature ${ }^{44}$. Dans cette optique, mais aussi pour contrer les opposants qui s'offusquaient du sang versé par les hommes, il était commun de souligner ses faibles dangers. Pour Oduaga, ils étaient "presque imaginaires", toujours affrontés avec prudence, sécurité, méthode "et c'est le sourire sur les lèvres et un calme orgueil dans le regard qu'il (le matador) étend à ses pieds, d'un coup d'épée, le monstre fougueux et mugissant". Les accidents n'étaient que le fruit de la témérité ou de la distraction. Cette opinion était celle d'Hemingway en 1932, de Théophile Gautier ou de Charles Davillier qui citait une phrase du torero Pepe Hillo (pourtant mort au combat !) : " notre art est arrivé aujourd'hui à un tel degré de certitude que nous traitons les taureaux avec autant de mépris que si c'étaient des moutons ". Ceux qui s'attardaient sur les morts étaient peu nombreux et beaucoup évoquaient avec humour des blessures souvent vite guéries ${ }^{45}$. Cette conception est quelquefois présente dans la seconde moitié du XXe siècle, Simone de Beauvoir traite le danger de "mythe" et Claude Popelin de légende, mais elle est devenue minoritaire bien qu'elle soit approuvée par des toreros célèbres. Dominguin considère que le matador ne pense pas plus à la blessure que l'automobiliste à l'accident, que la corrida se termine naturellement par la mort du taureau sans quoi il n'y aurait guère de volontaires. Ordonez affirme qu'il ne vit pas avec la peur au ventre, qu'il fait tout pour éviter le risque et qu'une blessure est une faute professionnelle ${ }^{46}$.

Cette conviction est confirmée par les statistiques. Si on examine les toreros ayant pris l'alternative et décédés au combat dans le cadre français et espagnol bien étudié par Bonifaz, trois phases apparaissent. De 1814 à 1911, il se produisit 20 décès, soit un tous les 4,9 ans. La période 1912-1947, de l'arrivée de Belmonte à la mort de Manolete, fut marquée par 15 décès, un tous les 2,4 ans soit presque deux fois plus qu'avant. Comme l'a bien vu Montherlant, cela correspondit à la transformation de la "corrida-combat" en "corrida-esthétique", avec un toreo proche de la bête et des taureaux transformés. Beaucoup de matadors eurent de la peine à s'adapter : Belmonte lui-même, l'initiateur de cette évolution, fut promis à une mort certaine par ses confrères et il reçut de nombreuses blessures; Manolete, inventeur du toreo de profil, fut tué par un taureau aféité (cornes épointées). De 1948 à 1994, le nombre de morts diminua nettement : 4, soit un tous les 11,75 ans. II serait souhaitable de rapporter ces décès au nombre de toreros en activité. Claude Popelin a estimé le taux de mortalité de tous les professionnels de la corrida (picadors, banderilleros..) à $4 \%$ pour un XIXe siècle élargi jusqu'aux années 1930 et à bien moins (sans préciser) pour la suite. Mais ces chiffres ne sont pas fiables : si les morts sont répertoriés, la liste exacte des toreros n'a jamais été dressée et des recherches récentes, portant sur le XIXe siècle, ont révélé de nombreux noms jusqu'alors inconnus, ce

43 Dans Tauromachie, art profond, 1951, p.14-15; Corrida, les lumières de l'arène, 1990, p.5-6; La peur, la beauté, la mort, 1981, p.15, 21-22, 117; L'envers de la corrida, 1955, p.64; La folie corrida, 1992, p.71; Toros, 19.03.1989, p.1; Corrida, la fin des légendes, 1992, p.23.

44 La corne et l'épée, 1908, p.9-10, 37; op.cit., p.2, 9-15, 21-22, 56. De même FOYE, Les courses de taureaux en France, 1890, p.13; L. FEUILLADE, op.cit. , p.91-92.

45 Idem, p.9-12; Mort dans l'après-midi (1932), éd. 1966, T.1 p.10051006, 1081; Voyage en Espagne (1843), éd. 1964, p.318; Voyage en Espagne (1862), éd. 1980, p.63. Blessures : H. MONTHERLANT, Bestiaires, op.cit., p.163; de même V. BLASCO IBANEZ, Arènes sanglantes, éd. 1921, p.143; E. HEMINGWAY, idem, p.1222.

46 La force des choses (1963), éd. Folio, T.2 p.74; Le taureau et son combat, 1981, p.64; Dominguin dans P. PICASSO, op.cit., p.10; Ordonez dans ZUMBIEHL, Des taureaux dans la tête, 1987, p.23-25, 79. 
qui modifie la base des calculs ${ }^{47}$. D'autre part, le nombre de corridas évolue et tous les toreros ne pratiquent pas avec la même intensité : un taux quelconque de mortalité ne représente pas le même danger s'il concerne un combat par individu et par an ou cent. II faut utiliser un coefficient de pondération, c'est-à-dire le nombre de taureaux combattus (6 par corrida) qui représentent autant de risque de mort. La courbe, établie d'après les statistiques bien connues des corridas en France et en Espagne au XXe siècle ${ }^{48}$, prouve que les décès se raréfient dans la seconde moitié du siècle alors que le nombre des bêtes s'accroît. Seize matadors furent tués pour 71469 taureaux entre 1901 et 1947, soit 1 pour 4467 et un risque de décès de 0,022\%; 4 morts eurent lieu de 1948 à 1993 pour 136134 taureaux, soit 1 pour 34033 ou $0,0029 \%$.

La régression de la dangerosité après 1945 résulte de phénomènes familiers aux aficionados, bien qu'ils aient rarement, et pour cause, effectué la corrélation : progrès de la médecine, mais aussi maîtrise du nouveau toreo, usage de la pique unique et prolongée qui épuise l'animal, fraudes diverses, apparues dans l'entre-deux-guerres, produisant des taureaux souvent trop jeunes, chutant, aux cornes aféitées ${ }^{49}$. II ne s'agit pas de nier le danger, mais les statistiques montrent que le discours sur l'omniprésence de la mort, voire une certaine égalité des chances, ne correspond pas à la réalité. Son développement depuis les années 1930-1950 s'effectue même dans un contexte inverse. On peut se demander pourquoi il s'est produit une confusion entre le but de la corrida (abattre un taureau dans les règles de l'art) et le risque inhérent (la mort de l'officiant). Comme le signalait Dominguin ${ }^{50}$, il y eut plus de morts en courses de Formule 1 (18 de 1950 à 1994, plus 7 en essais officiels), peut-on néanmoins avancer que leur finalité soit de tuer les pilotes?

La survalorisation de la mort provint de trois courants différents, mais convergents. Le fait majeur réside dans cette conversion à la corrida de certains intellectuels des années 1920-1930, alors que ce milieu était jusqu'alors hostile. Au même moment, en Espagne, le groupe formé autour de Belmonte en 1923 ainsi que la "génération 1927" rompirent avec le discours traditionnel centré sur le nationalisme, la gloire, la bravoure. Français et Espagnols développèrent des conceptions proches, multiformes, dont on ne peut donner ici que les grands traits. La corrida fut transformée en art vivant, sublime, parce que spontané et éphémère, en rite religieux, sacrificiel, en lieu symbolique de destruction de l'ordre bourgeois, car elle permettait de communier avec le peuple et de libérer les émotions contraintes par la morale, ce dernier aspect étant spécifique aux cénacles français. Alors qu'en Espagne, Lorca et Bergamin minoraient la violence et le folklore populaire pour rendre la corrida acceptable hors des frontières, Montherlant, Bataille, Leiris évoquaient ce qui était jusqu'alors repoussé avec horreur en leur pays : la lutte des corps, les passions, les désirs et les instincts les plus refoulés, la violence, la jouissance qui, à leurs yeux, se déchaînent dans le combat, parmi le public, et qui font de la corrida un rite exutoire, un moment d'explosion des éléments troubles de l'homme.

Cet amoralisme affiché libéra une lecture érotique où la mort était présente. Montherlant, l'un des premiers en 1926, Bataille, qui assimilait la jouissance à une petite mort, Leiris ou Picasso considérèrent que les passes, l'éventration du cheval, l'agonie du taureau symbolisaient le rapport sexuel. Cette lecture s'effectuait en priorité sur l'animal, mais elle concernait aussi le torero lors de la cogida (soulèvement par les cornes) ainsi mise en valeur. La recherche de l'homme vrai, le rejet de la morale, produit de l'ordre bourgeois, alors que les opposants à la corrida évoquaient un progrès issu des Lumières, justifia aussi la revendication ouverte, délibérée du sadisme. Montherlant, l'un des

47 J. BONIFAZ, Victimas de la Fiesta, Madrid, Espasa Calpe, 1991; H. MONTHERLANT, Coups de soleil, éd. 1976, p.212; C. POPELIN, op.cit., p.64; F. LOPEZ IZQUIERDO, Plaza de toros de la Puerta de Alcala, 1739-1874, 1985.

48 Pour l'Espagne, nous utilisons les chiffres de la Gran Encyclopedia d'Espana, Madrid, 1990, T.7, art. "corrida". On trouve une liste analogue, à quelques unités près, dans B. BENNASSAR, op.cit., p.206. Pour la France d'avant 1951, il s'agit d'estimations d'après D. GATUMEL, op.cit., graphiques et A. LAFRONT, op.cit. Après 1951, la revue Toros publie régu lièrement les chiffres (cf. 26.08.1988). Par contre, il est quasiment impossible d'établir les fré quences précises des autres spectacles: novillades, becerrades, etc.

49 Voir J.P. DARRACQ, "Un demi-siècle de mensonges taurins", Toros, 18.12.1988, p.1-7; M. ROUMENGOU, Fraudes sur les taureaux de combat, 1977.

50 Dans F. ZUMBIEHL, op.cit., p.49. 
premiers là aussi, aimait s'attarder sur le sang, l'étripage du cheval, la mort du taureau qui laissaient s'exprimer la sauvagerie de l'homme. Dans les années 1930, Picasso produisit de nombreuses scènes du premier tercio, notamment des éventrations de chevaux, alors que le caparaçon, qu'il détestait parce qu'il affadissait le combat, était en usage depuis 1928. Mais le sadisme ne se rapportait pas seulement à l'animal. L'année (1922) où il découvrit la corrida, Bataille assista à la mort de Granero par défoncement d'un oeil. L'événement le fascina, lui procura un plaisir trouble, né de son horreur et de son intolérable beauté : " Jamais, dès lors, je n'allais aux courses de taureaux sans que l'angoisse ne me tendît les nerfs intensément. L'angoisse en aucune manière n'atténuait le désir d'aller aux arènes. Elle l'exaspérait au contraire, composant avec une fébrile impatience. Je commençais à comprendre que le malaise est souvent le secret des plaisirs les plus grands". Enfin la corrida était le reflet de la condition humaine. Pour Montherlant ou Leiris, le combat de l'homme était l'occasion de se mettre en péril pour prouver sa maîtrise du destin. Bataille et Leiris considéraient qu'elle symbolisait le sort de l'homme, la mort étant sans cesse présente derrière la vie. L'ambivalence du rite, soulignée aussi par Picasso, où la beauté et le faste se mêlent au danger, au sang, à la mort, leur semblait renforcer la tragédie ${ }^{51}$.

S'il était déjà bien constitué, ce discours sur la mort n'occupait pas encore une place prépondérante. Des intellectuels convertis à la même époque ne l'évoquaient guère : Hemingway (dont l'ouvrage Mort dans l'après-midi, 1932, véritable manuel de tauromachie, ne concerne pas l'homme, contrairement à ce que l'on croit spontanément, mais le taureau), Sartre et Simone de Beauvoir gardèrent la conception traditionnelle de la domination de l'homme sur la bête ${ }^{52}$. Dans le contexte révolutionnaire de la décennie 1930, Lorca donna la priorité à la fusion avec le peuple. Jusqu'aux années 1960, Picasso représenta peu de toreros, préférant le premier tercio. Deux facteurs propulsèrent ces propos au premier plan après 1945. Dans la première moitié du XXe siècle, un nouveau toreo se développa, fondé sur une certaine immobilité du matador, la multiplication des passes pour maîtriser la vitesse de l'animal, lui imposer un passage au plus près de l'homme et un retour immédiat pour enchaîner le mouvement suivant. Dans ce jeu, qui remplaçait le mode traditionnel fondé sur l'esquive, la défense, la mobilité, l'homme semblait retenir le danger et la mort pour jouer avec eux dans un ballet incessant ${ }^{53}$. Or nous avons vu que la période 1912-1947, celle de l'apprentissage de cette "corrida esthétique ", fut la plus meurtrière de l'époque contemporaine. Cette situation crédibilisa l'impression donnée par le spectacle ainsi que le discours sur la condition humaine. Le mythe naquit d'une certaine réalité. II put se répandre grâce à la généralisation de ce nouveau combat après la guerre, bien que les fraudes et les manipulations génétiques aient rétabli et accentué la sécurité antérieure en produisant des taureaux aux attitudes prévisibles, aux cornes resserrées et épointées, amoindris en taille ou en âge et sévèrement châtiés à la pique.

Le second facteur intervint après 1945. Dans un contexte marqué par la triste expérience de la guerre, les aspects les plus sulfureux du discours d'avant 1939 (l'amoralisme, le sadisme, la recherche de l'homme vrai (Bataille) ou du surhomme (Montherlant)), furent minorés par les nouvelles générations artistiques et intellectuelles au profit de l'esthétisme, du sacrifice religieux et du risque de mort, celui-ci profitant de l'interrogation sur la condition humaine menée, par exemple, par la philosophie existentialiste ou la littérature de l'absurde. Cocteau illustre bien ce glissement. Ses écrits évoquèrent l'art, la métaphore sexuelle et la tragédie : la corrida était une noce où l'homme et le taureau, ambassadeur de la mort, s'attiraient et se craignaient, s'aimaient et se rejetaient dans la succession des passes. Le torero éloignait la "Dame blanche" en tuant l'animal, mais elle gagnait

51 J. BERGAMIN, op.cit., p.35-59, 88-95; F. GARCIA-LORCA, op.cit. , T.1 p.918-930; H. MONTHERLANT, Bestiaires, op.cit., p.82-83, Va jouer avec cette poussière, 1966, p.68-69, 189-190; P. SIPRIOT, op.cit., T.1 p.18-19, 38, 66, 101-110, 149-151, T.2 p.32-45, 149, 246-262, 295; M. LEIRIS, Miroir.., op.cit., p.25-39, 48-67, L'âge.., op.cit., p.70-75; L. BOYER, Michel Leiris, Ed. Universitaires, 1974, p.49-50, 66-81; G. BATAILLE, Oeuvres, T.1, p.50-53, T.12 p.187, 272 et "A propos de Pour qui sonne le glas", Actualités, L'Espagne libre, 1946, p.120 (citation); M. SURYA, Georges Bataille, Séguier, 1987, p.57-71, 175, 192, 290-293; Picasso.., op.cit. , p.12-15, 23-49, 97, 119-127, 147-169, 230.

52 Op.cit., T.1 p.1000, 1005, 1062, 1172-1177, 1196; Lettres au castor, 1982, T.1 p.250; op. cit., T.2 p.73-74.

53 G. LESTIE, Règles et secrets de la corrida, éd. 1964. 
toujours, quelquefois dans l'arène ou à la fin de la vie ${ }^{54}$. C'est à cette époque que le Chant funèbre pour Ignacio Sanchez Mejias (1934) de Garcia Lorca, à l'origine un poème de circonstance pour le décès d'un ami, devint le manifeste de cette nouvelle sensibilité, sans cesse cité, réédité, inspirant les artistes (orchestration de Maurice Ohana en 1950).

L'autre courant de valorisation du danger provint des matadors eux-mêmes. Dans les années 1910, Belmonte rompit avec leur image traditionnelle (fêtes, flamenco, conquêtes féminines), se lia avec des cercles artistiques madrilènes, puis avec Montherlant, et lut beaucoup pour se donner une image de torero des intellectuels. C'est alors qu'il adopta et rapprocha le mot d'ordre de D'ANNUNZIO ("le danger est le centre de la vie sublime") du toreo de près qu'il inventait au même moment, insistant sur l'angoisse, la peur, le trépas, l'analogie avec l'amour. L'évocation du risque de mort n'était pas vraiment nouvelle : Cuchares (1818-1868) affirmait quelquefois que la meilleure suerte (série de passes) était celle qui lui permettait de rentrer chez lui. Mais il revint à Belmonte de l'insérer dans une vision d'ensemble, de la dramatiser par le nouveau toreo et de la transmettre aux intellectuels. Depuis, les matadors, dont les plus célèbres fréquentent les milieux artistiques, intellectuels ou mondains ${ }^{55}$, hésitent souvent entre leurs convictions personnelles (qui leur fait accepter l'esthétisme, la collaboration avec le taureau, mais refuser la sensualité, jugée trop proche de la bestialité, et l'héroïsme suicidaire) et ce discours très porteur dans une société qui bannit progressivement la mort de sa vie quotidienne, mais se plaît à la regarder en des endroits précis, sorte de lucarnes du voyeurisme. II est ainsi devenu le principal argument publicitaire lors de la carrière (1960-1970) d'El Cordobes, la plus médiatisée et la plus populaire du siècle. Son impresario lui fabriqua une image, vite véhiculée par les médias, de jeune contestataire de l'ordre établi, bien qu'il fût proche de Franco, flirtant avec la mort par ses fanfaronnades et ses acrobaties, alors qu'il combattait souvent des bêtes de petite taille, d'où les slogans "L'homme au plus près de la mort" ou "Seul face au danger", un film, L'Apprentissage de la mort, une biographie, Où tu porteras mon deuil (1967) de Lapierre et Collins ${ }^{56}$.

Le dernier courant de promotion du mythe fut constitué par les aficionados qui s'en servirent plus ou moins consciemment pour profiter de l'attrait suscité par ce discours et pour endiguer un mouvement contraire des sensibilités. L'époque contemporaine est en effet marquée par une évolution des attitudes vis-à-vis du monde animal, un phénomène révélé par quelques travaux pionniers ${ }^{57}$, mais encore mal connu dans son développement historique exact et ses motivations, qui se traduit notamment par l'intérêt pour les animaux de compagnie, le refus croissant des mauvais traitements (du moins ceux perpétrés en public), la volonté de protéger les espèces sauvages. Or la corrida illustre ce processus souvent lent et inconscient. Le développement du tourisme en Espagne au XIXe siècle profita au spectacle tout en créant un facteur inattendu de transformation. Devant la réprobation croissante des étrangers, l'usage de lâcher les chiens sur les taureaux peu belliqueux fut abandonné vers 1860 , celui de la media-luna (pique en croissant de lune pour couper les jarrets) en 1880 et les combats d'animaux cessèrent au début du XXe siècle. En 1928, le dictateur Primo de Rivera, qui voulait donner de l'Espagne une image de pays moderne et civilisé, rendit le caparaçon obligatoire pour le cheval, afin d'"éviter ces horribles spectacles qui répugnent tellement aux étrangers et aux touristes ". Les banderilles de feu furent supprimées par la suite (règlement de 1962). À chaque fois, ces changements furent violemment refusés par les aficionados qui vitupérèrent les inventions des âmes sensibles, crièrent à la mascarade et à la dégradation des principes toniques et virils de la

54 "La Dame blanche", Nouvelle Revue française, avr. 1957, p.577-594.

55 Dans les années 1950-1960, Dominguin sympathisa avec Picasso, Cocteau, Leiris, Ava Gardner, Sophia Loren.

56 M. CHAVES NOGALES, Juan Belmonte, 1990, p.158-161, 201-208, 215-219; H. MONTHERLANT, Va.., op.cit., p.66-69, 217; F. ZUMBIEHL, op.cit., p.8-22, 30, 45-49, 75 (toreros); B. BENNASSAR, Histoire de la tauromachie, op.cit., p.124-125 (Cordobes).

57 M. AGULHON, "Le sang des bêtes. Le problème de la protection des animaux en France au XIXe siècle", Romantisme, 1981, p.81-109; V. PELOSSE, "Imaginaire social et protection de l'animal. Des amis de l'an $X$ au légis lateur de 1850", L'Homme, 1981, 4, p.5-33, 1982, 1, p.33-51. Ce chantier se développe, voir K. KETE, The beast in the boudoir : Petkeeping in 19th century Paris, U. California Press, 1994. 
corrida $^{58}$.

C'est alors qu'ils développèrent deux défenses, à la fois vécues comme des réalités et utilisées pour préserver le combat. D'abord, à partir des années 1920, ils minimisèrent, voire nièrent la souffrance des bêtes ${ }^{59}$. Puis leur acceptation des réformes (étant eux-mêmes soumis à cette évolution des sensibilités mais avec un écart chronologique sensible : les décennies 1960-1970 pour le caparaçon $^{60}$ ) les obligea à soutenir que les spectateurs ne venaient pas voir la douleur et le sang, mais participer à un rite ${ }^{61}$. L'autre discours, plus fréquent, fut celui du danger pour l'homme et d'une certaine égalité des chances. Un thème qu'ils n'inventèrent pas, mais qu'ils reprirent dès les années 1950 en triant eux aussi dans le discours intellectuel initial (la sexualité, l'amoralisme, le sadisme étant le plus souvent refoulés) et qu'ils diffusèrent largement pour légitimer la corrida en réfutant les accusations de cruauté ou de combat inégal et pour masquer une réalité de plus en plus contraire. Cette stratégie a bien réussi, puisqu'elle a bloqué toute évolution depuis 1928 et imposé le mythe comme une évidence, aidée en cela par une certaine adhésion des élites.

\section{Pour conclure : la diffusion du mythe dans l'opinion publique}

Retracer la construction d'une légende ne suffit pas pour comprendre comment celle-ci s'est diffusée dans l'opinion publique jusqu'à devenir une image d'Épinal de la corrida. Nous n'indiquerons ici que les grandes lignes du processus en renvoyant à ce que nous avons écrit ailleurs. À peine ébauchée dans l'entre-deux-guerres, la conversion d'une partie des élites nationales s'amplifia dans les décennies 1950-1960, touchant non seulement les artistes et les intellectuels, mais aussi le monde politique, la magistrature et l'Église catholique. Cette évolution s'inscrit dans un mouvement contemporain de détachement de ces groupes dirigeants vis-à-vis du monde environnant dont l'histoire reste à écrire, mais qui s'illustre, par exemple, dans le succès de l'idéologie de la maîtrise de la nature (grands travaux, barrages, agriculture intensive..) ou dans une certaine marginalisation des associations de protection durant les années $1950-1960^{62}$. Cette tendance à moyen terme allait à l'encontre de l'orientation pluri-séculaire dont nous avons parlé, tout en la prenant en compte. Car, làencore, les aspects les plus sulfureux des discours pionniers de l'entre-deux-guerre furent délaissés au profit des plus adaptés à la sensibilité ambiante, des plus acceptables par l'opinion publique : l'esthétisme et la tragédie.

La conversion des élites permit l'investissement des médias nationaux par les aficionados (après les régionaux au début du siècle). C'est ainsi que, dans les années 1950-1970, Auguste Lafront livra des billets à France-Soir, Jean Eparvier à L'Equipe, Jean Cau à L'Express, Yvan Audouard au Canard Enchaîné, Marcelle Auclair à Marie-Claire, etc. L'exemple du journal Le Temps, hostile à la corrida jusqu'à son sabordement en 1942, montre bien cette évolution : sa succession, organisée par Beuve-Méry en changeant le titre (Le Monde), mais en gardant la même équipe, s'accompagna d'un revirement d'attitude sous l'impulsion d'Olivier Merlin, jeune reporter au Temps, devenu chef du secrétariat de rédaction du Monde, qui fit entrer la corrida dans la rubrique "sport" dont il était le responsable ${ }^{63}$, avant de donner la plume aux aficionados, tel Jean Lacouture à partir de 1962. Cette

58 L. FEUILLADE, op.cit. , p.89-90; F. BERTRAND, De Biou y Toros à Toros, évolution, action et influences d'une revue tauromachique, Maîtrise, Montpellier 3, 1992, p.92-94 (les revues taurines); E. FORNAIRON, op.cit., 1955, p.16-20. Même refus chez Montherlant, Picasso, Hemingway. Rivera cité par E. HEMINGWAY, op.cit., T.1 p.993.

59 H. MONTHERLANT, Bestiaire, op.cit. , p.101; " Courrier des lecteurs ", Le Monde, 06.10.1986, p.30.

60 c. PELLETIER, op.cit., p.112; Toros, 18.12.1988, p.5.

$61 \mathrm{~J}$. CAU, La folie corrida, 1992, p.72; M. LAGIER-AYMA, Tauromachie, la fête, l'art et le rite, 1991, p.159, 175.

62 Voir J. LALOUETTE, "Vivisection et antivivisection au XIXe siècle", Ethnologie française, XX, 2, 1990, p.156-165; E. BARATAY, L'Eglise et l'animal (France, XVIle-XXe siècles), Cerf, 1996. Sur les élites et la corrida : E. BARATAY, E. HARDOUIN-FUGIER, op. cit., chap.4.

63 E. SABLIER, La création du Monde, Plon, 1984, p.43-52, 71. Voir Le Monde, 5-9 Mai 1949. 
situation perdure de nos jours, puisque la quasi-totalité des articles consacrés à la corrida est le fait de ses partisans.

Ces deux phénomènes aidèrent à sa transformation en phénomène de société dès les années $1950^{64}$ et ils assurèrent la marginalisation des adversaires qui perdirent tout véritable accès aux médias contrairement à l'Espagne où le débat est ancien et naturel. Sans opposition réelle, le mythe put se déployer à son aise, s'imposer à l'opinion publique, être vécu par les aficionados, selon les individus et les thèmes, comme une vérité ou comme une erreur tolérable au regard de ses pouvoirs de légitimation, la réalité, lorsqu'elle est connue, étant souvent réservée aux magazines à faible diffusion, destinés aux plus avertis.

Ainsi apparaissent les deux fonctions du mythe : il a permis d'imposer la corrida dans un pays majoritairement hostile $\left(83 \%\right.$ en $1993^{65}$ ), supportant de moins en moins la violence publique envers l'animal; il conforte maintenant une sociabilité méridionale qu'il a lui-même contribué à construire. Bel exemple de reconstruction du passé où l'histoire est mise au service d'une finalité sociologique.

64 Films (Sang et lumières, 1953; Les Châteaux en Espagne, 1954), chansons (C. Aznavour, Toréador, 1964), croissance de l'édition depuis la décennie 1970, retransmissions télévisées sur Canal+ depuis 1985.

65 Sondage L. Harris dans C. LAURENT, "L'arène comme lieu de mémoire : la corrida à l'épreuve de l'histoire", Des bêtes et des hommes, C.T.H.S., 1995, p.297. 